\title{
Network Analysis Methods to Measure Sociometric Status
}

\author{
Simona Gozzo, Venera Tomaselli* \\ Department of Political and Social Sciences, University of Catania, Italy
}

Copyright $(2016$ by authors, all rights reserved. Authors agree that this article remains permanently open access under the terms of the Creative Commons Attribution License 4.0 international License.

\begin{abstract}
This paper proposes an innovative methodological approach to measure sociometric status in small groups of pupils. Although it uses indirect data collected by interview, in this study the sociometric status is analysed by direct observation. This method is specifically suitable when the target population concerns pre-school children. Their cognitive competence, in fact, is not as well developed as their relational abilities. Hence, the indicators constructed are more reliable than the measures derived by the subjective perception of interviewed pupils. The Network Analysis methods allow for the definition of sociometric status by means of regular equivalence. Employing lambda sets and cliques, then, we specify further roles into distinctive small groups. The results show that sociometric status can be revealed by regular equivalence. Besides, the Network Analysis approach allows for the observation of further relational skills, not strictly associated with traditional social roles, detectable only through lambda sets and cliques.
\end{abstract}

Keywords Sociometric Status, Role Classification, Structural Equivalence, Lambda Sets, Cliques

\section{Introduction}

This paper describes and analyses relational patterns among pupils at an infant school, in order to classify roles into distinctive groups by means of Network Analysis (NA) measures.

Several studies have shown that peer relationships have significant effects on language development, learning abilities and empathetic capabilities $[1,2]$. To contextualize the research, a recent study [3] characterises selection and socialization aspects of school-age children and adolescent peer groups. Using nearest neighbour observations, affiliative subgroups are identified. Secondly, additional behavioural observations and sociometric data are used to show that high mutual proximity subgroups reflect friendship relations, whereas low mutual proximity subgroups reflect common social goals without subgroup dyadic ties.
Nowadays, infant behaviour cannot simply be studied in relation to adults. Parents continue to play central roles in the construction of the child's identity but the child's world becomes progressively more polycentric. Relationships with peers, therefore, are as important as those with parents. On the basis of this assumption, our study explores the capability of pre-school 3-5 years children to relate with peers in the construction of social roles.

The concept of role suggests a structure of relationships among the subjects in a network. In a NA approach, the notion of social role depends conceptually, theoretically and formally on the specific relationships that link the set of actors and their positions across the network.

Our research was carried out in an infant school, observing 42 pupils aged from 3 to 5 years during the whole month of October and the first three weeks of December. The collected data refers to seven waves. In each wave, the teachers observed and recorded the pupils' behaviour on Saturday when the same pupils all play together in a common space with no difference in class or age. Finally, it was possible to compare children who continuously occupy the same subgroup, maintaining the same sociometric status, as well as children who change their relational behaviour.

\section{Theoretical Framework}

In our hypothesis, children in pre-school age (3-5 years old) have relational skills that are sufficiently developed to allow for the analysis of social roles $[4,5]$. In addition, the paper proposes a parallelism between analysis of social roles and sociometric status, implementing this through NA. Following NA approach, the concept of role is defined in terms of equivalence between the actors within a network. Individuals play the same role if they are similar, taking into account the number and type of relationships they have with others. The concept of sociometric status refers to the degree to which the children like or do not like the other children of their groups. That is to say, it refers to the level of satisfaction in the peer group. Sociometric tests are usually applied to measure the attraction and repulsion between persons. Attraction and repulsion are considered orthogonal processes in social relations, sociometric status then affect both how individuals perceive others and how 
others perceive the individual. The experience of peers may be understood as the product of three distinct forces, acting on the interpersonal level: attraction, repulsion, and indifference. From this theoretical basis, many scholars have contributed to the development of the sociometric method.

Many authors, through the interview method, have found that the construction of sociometric status $[6,7,8,9,10]$ occurs in children aged 5-12 [11, 12, 13]. In this study, by contrast, sociometric status is measured by direct observation. This method is suitable for obtaining consistent data in pre-school children, given the remarkable gap that is found between verbal and relational ability at that age. For school-age children and adolescent groups, the direct observation method can be used with some form of self-reporting $[14,15]$. In the case of pre-school children, only direct observation is reliable as a source of network information [16]. The method usually entails watching children many times for several days or weeks, and recording their interactions in terms of who is active or passive in creating an affiliative social relationship.

In spite of the fact that the methods used and the age groups are so different, similar findings emerge, as in previous studies carried out using interviews $[6,17]$. These studies identified the following types of sociometric status:

1. popular: altruistic children, who comply with social norms, have high social impact and preference (i.e. many contacts and many positive relationships)

2. rejected: excluded by other children because of their aggressiveness or self-isolation (they have little or no contact and negative relationships)

3. controversial: aggressive, in spite of the fact that they define themselves as popular, avoiding peer rejection and isolation (high social impact and high index of aggressiveness)

4. average: $60 \%$ of interviewed pupils fall into this category. They are less friendly than the popular but more sociable than the rejected

5. neglected: usually with only a good relationship, often repeated, with the same subject (best friend).

The studies of sociometric status focused on two scores: acceptance and rejection, defined by the number of the most liked and least liked nominations. Combining these two kinds of nomination, 'social preference' and 'social impact' indicators are obtained. Social impact is the standardized sum of acceptance plus rejection scores for each pupil. Social preference is the standardized difference between acceptance and rejection scores [4].

We define social impact and social preference as indicators, even if we do not employ the nominations. In our research, positive and negative occurrences correspond to pros or cons of actual behaviour. For this purpose, actions are considered. We use these attributes of the children to evaluate the different relational propensities, specifically those referring to aggressiveness rather than to sociability. Further attributes of the pupils are: gender, age, origin (namely if, in previous school years, children were already in classroom settings or not). Finally, the results of ego-network analysis are also used as attributes of children where ego is each pupil in the whole network. The ego-network is therefore based on the ties that, directly and indirectly, refer to each ego/subject.

Furthermore, we do not use the controversial profile since we observe aggressiveness or sociability as behaviour, avoiding the investigation of subjective perceptions.

\section{Material and Methods}

We have chosen to observe pre-school pupils, since they are on the verge of starting their first social experiences with peers. Children aged 3-5, in fact, have a low number of pre-existing relationships, and so are less affected by previous socialization experiences [18].

The main aim of our study is to classify social roles by means of NA. We employ structural equivalence, cliques and lambda sets in order to specify the role of each child within the whole network, identifying clusters on the basis of the specific relational abilities of popular, broker and/or embedded children. We then use ego-network measures and other features of children to identify the significant traits for each ability.

Data collected from observed behaviour are recorded in a matrix for each week of October and December. Afterwards, we overlap seven weighted and directed adjacency matrices in order to build the whole network for each month. Applying NA methods to these matrices, we highlight a pattern of relationships among the children.

The months of October and December were chosen because school starts in October, and some children have their first impact with a school setting in that month. In December, instead, a Christmas performance is being staged and all the children are involved in a common project. We hypothesise that this circumstance could increase the propensity to relate with others. Besides, taking an overview of the whole network, children ('nodes', in NA language) rarely maintain stable relationships. Despite this, an interesting 'emergent effect' [19] is observed. The time and quality involved in the relationships influence the increase of stability both for the structure of the network and the roles played by children.

\subsection{Measuring Relational Dynamics}

The analysis of sociometric status necessarily involves the introduction of the concept of equivalence, as used in NA distinguishing in structural [20], automorphic [21] and regular equivalence. Each method has different underlying assumptions concerning the identity of related subjects. Structural equivalence expects that two actors play the same role if they are associated with identical links to other actors. Automorphic equivalence defines two actors as equivalent if they have the same relational structure with other nodes, 
even if these nodes are not the same actors [21]. Finally, regular equivalence refers to a more general concept of 'similarity': two nodes are equivalent when they have links with the same profile of actors. Specifically, regular equivalence defines clusters of pupils, where each group is homogeneous with respect to the type of relationship. The definition requires that "equivalent actors have equivalent sets of alters" [21]. Regular equivalence is the most flexible measure and for this reon, it is widely used to identify general social roles in a network [23].

NA methods are suitable for analysing different aspects of relational skills. Regular equivalence, indeed, allows studying the relational behaviour of each child, distinguishing between the most and least sociable profiles. Further information about relational dynamics is derived from lambda sets and cliques. Specifically, the ability to play the function of intermediate is measured by lambda sets and the incidence of cohesive subgroups by the clique.

In order to identify the sociometric status of the nodes in a network, we employ regular equivalence applying the REGE algorithm [24], the most suitable for directed and weighted matrices.

According to White and Reitz [24], regular equivalence for single-relation networks is defined as:

if $\mathrm{G}=<\mathrm{P}, R>$ and $\equiv$ is an equivalence relation on $P$, then

$\equiv$ is a regular equivalence if and only if for all a, b, c $\in \mathrm{P}$ $a \equiv b$ involves that:

1. aRc implies there exists $d \in P$ such that $b R d$ and $d \equiv$ $c$

2. $\quad c R a$ implies there exists $d \in P$ such that $d R b$ and $d \equiv$ $c$.

REGE is an iterative algorithm. Within each iteration, a search is implemented to optimize a matching function between nodes or vertices $i$ and $j$. As a consequence, for each $\mathrm{k}$ in i's neighbourhood the algorithm searches for an $\mathrm{m}$ in j's neighbourhood of similar value. A measure of similar values is based upon the absolute difference among sizes of ties. The measure is then weighted by means of the degree of equivalence between $\mathrm{k}$ and $\mathrm{m}$ at the previous iteration. The matching is thus optimized. This is summed for all members of i's neighbourhood over all relations and normalized to provide the current iteration measure of equivalence between $i$ and $j$. The procedure is repeated for all the pairs of vertices for a fixed number of iterations.

The result of this iterative procedure is a similarity matrix, which provides a measure of regular equivalence. This matrix is then submitted to a clustering routine using the single hierarchical linkage. We thereby obtain a dendrogram of the regular similarity measure with the level at which any pair of actors are aggregated [24].

In our analysis, however, the data collected during the first month (October) does not allow for the use of this method, since, in the first stage of socialization, repeated relationships are not widespread enough within the network.
As a consequence, we measure the similarities between nodes in the network, comparing empirically the profiles of rows and columns in the similarity matrix (Figure 1). Since the matrix is weighted, similarity is evaluated via the covariance among the nodes.

$\begin{array}{ccccccccc} & u_{1} & u_{2} & u_{3} & u_{4} & u_{5} & u_{6} & \ldots & u_{n} \\ u_{1} & - & 0 & 0 & 0 & 0 & 1 & \ldots & \ldots \\ u_{2} & 1 & - & 1 & 1 & 1 & 1 & \ldots & \ldots \\ u_{3} & 1 & 1 & - & 1 & 1 & 1 & \ldots & \ldots \\ u_{4} & 0 & 0 & 0 & - & 0 & 1 & \ldots & \ldots \\ u_{5} & 0 & 1 & 0 & 0 & - & 0 & \ldots & \ldots \\ u_{6} & 0 & 1 & 0 & 0 & 0 & - & \ldots & \ldots \\ \ldots & \ldots & \ldots & \ldots & \ldots & \ldots & \ldots & - & \ldots \\ u_{n} & \ldots & \ldots & \ldots & \ldots & \ldots & \ldots & \ldots & -\end{array}$

Figure 1. Similarity matrix $u_{i} \times u_{i}$.

The similarity matrix and REGE algorithm are applied to cluster children identifying social roles that are suitable to be compared in terms of sociometric status. This is the main goal of our study. In addition, by means of NA procedures, we are able to further specify social and relational skills.

The ability to be mediators in a relational context is measured by the lambda set, based on the assumption that the members of a cluster have greater edge connectivity with other members than with non-members. Given a graph $\mathrm{G}(\mathrm{V}, \mathrm{E})$, a lambda set $S$ represents the edge-connectivity of two vertices $\boldsymbol{a}$ and $\boldsymbol{b}$ in the graph. It is a subset of $V$, such that for all:

$$
\mathrm{a}, \mathrm{b}, \mathrm{c} \in \mathrm{S} \text { and } \mathrm{d} \in \mathrm{V}-\mathrm{S} \text {, then } \lambda(\mathrm{a}, \mathrm{b})>\lambda(\mathrm{c}, \mathrm{d})
$$

A lambda set is a maximal subset of vertices with the property that the edge connectivity of any pair of vertices within the subset is strictly greater than the edge connectivity of any pair of vertices, one of which is in the subset and the other is outside [25]. We can characterize a lambda set $S$ by the minimum connectivity among its members, satisfying the axioms of an ultra-metric similarity measure. In this sense, computing a lambda set, we can obtain a matrix of partitions in which a value of $K$ in an $i$-column and in a $j$-row indicates that the node $j$ is in the $k$-partition and the other members of the partition form a lambda set with minimum edge-connectivity. This information allows deriving a permutation of the nodes used in a particular hierarchical clustering of the nodes in a network, properly corresponding to a lambda set [25].

This technique, then, is employed to define homogeneous sub-groups based on the ability to be intermediaries, ranking the nodes of the network. In particular, if we remove nodes with the highest scores, the whole structure of the network substantially changes. By contrast, nodes with median scores have the ability to act as intermediaries but their role does not affect the shape of the network. These latter nodes are mainly important for the relational dynamics between two or more subjects or small sub-groups.

Finally, a clique is the maximum complete sub-graph 
composed of three or more nodes. It is, therefore, a maximal subset of nodes in which each node is directly connected with each other [26]. A clique $S$ of a graph $G(V$, $E$ ) is a maximal subset of $V$ such that for all:

$$
\mathrm{S} \in \mathrm{S}, \alpha(\mathrm{s}, \mathrm{S})=|\mathrm{S}|-1
$$

In the case of directed graph, the procedure for the analysis of cliques - i.e. the identification of the structure of the cohesive partitions within the networks - should only take into account mutual links (strong cliques). The direction of ties can be ignored (weak cliques) since these only refer to the presence or absence of a relationship. The same node or set of nodes may belong to different cliques, also overlapped. It, in fact, has a score that corresponds to the number of times that a node is present in the identified cliques. At the structural level, collective ability to organize complex relational structures, beyond the dyadic relationship, is also shown $[26,27]$.
Since this study is aimed to identify the propensity of each pupil to be part of a group, the cliques are useful to describe relational abilities.

\section{Results}

\subsection{The Analysis of Social Roles}

The identification of roles is defined on a monthly basis. Looking at the two graphs, we can see that in October (Figure 2A) pupils are less connected with each other. During the first month of school, in fact, socialization is starting and there is a lack of repeated and important ties among children. Then, we aggregate children employing the Clauset-Newman-Moore algorithm, implemented in Nodexl [28]. This algorithm looks at how much the vertices are connected to one another.

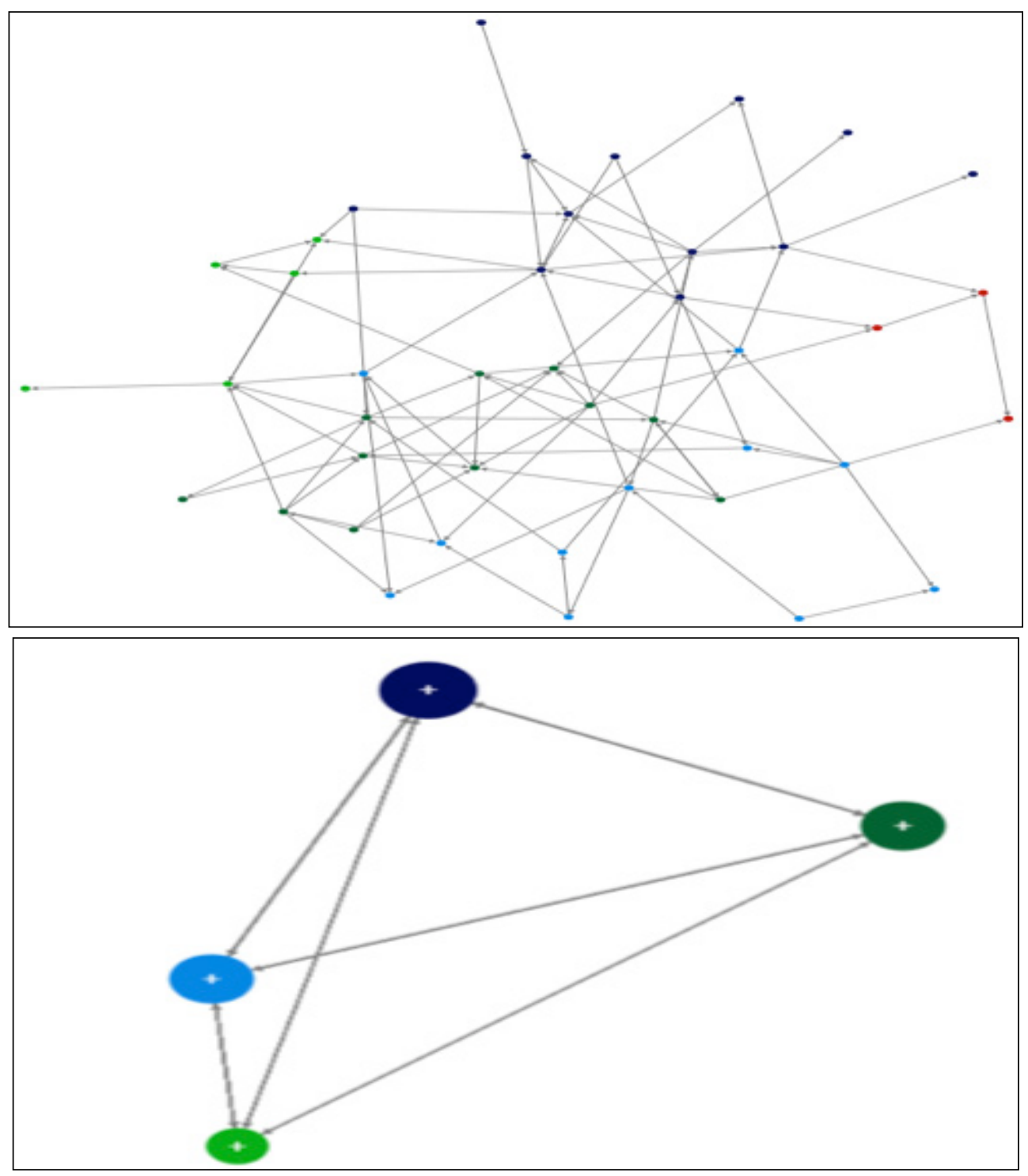

Figure 2A. Graphs of cumulative matrices and clusters for October data. 

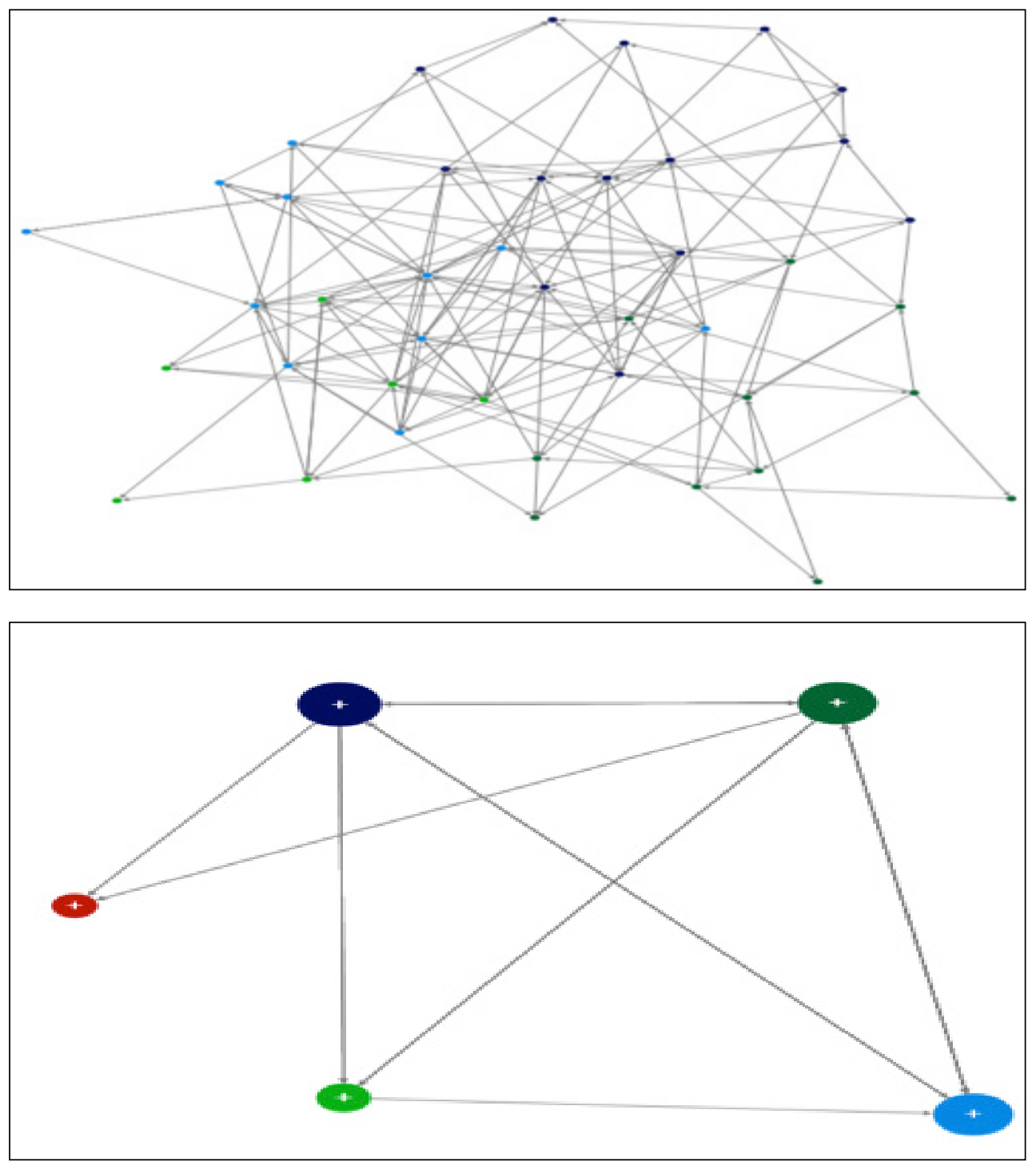

Figure 2B. Graphs of cumulative matrices and clusters for December data.

We notice that in December (Figure 2B) the number of groups is reduced but their shapes are similar with the same ties among clusters. Nevertheless, the expanded graphs show more ties in December with an increasing reciprocity and a denser net than in October.

With the aim of identifying the presence of different types of roles within the net, regular equivalence, lambda sets and cliques procedures are employed. Firstly, using regular equivalence, the October data show social roles based on the individual propensity to contact and/or to be contacted by others. Specifically, the following profiles of children are identified:

1. isolated, without relationships (similar to the rejected as defined in literature)

2. invisible, with only one tie (similar to the neglected)

3. average, with a high index of aggressiveness in October

4. popular, with a special propensity to contact and/or be contacted.
The isolated children are only contacted by others. These children are not, therefore, actually isolated but rather tend to self-exclusion. Despite the fact that these pupils are defined as neglected or rejected in the literature, in this study they belong to the network. Since the research is based on direct observation, we hypothesise that the condition of rejected has greater persistence as cognitive trait or subjective perception than as relational pattern. Applying the interview, indeed, only few children choose these pupils as friends. Instead, the data collected by means of direct observation and processed by NA methodology highlight that the rejected child is contacted by other children and, then, he is not necessarily refused but he only seldom replies to the contact.

Besides, self-exclusion decreases over time and in December isolated and invisible children lie in the same group. In December, the data structure permits to use the criterion of regular equivalence, applying the REGE algorithm, the most suitable according to the nature of the data. The REGE algorithm distinguishes three types of nodes, 
defined in the literature as: sinks, with only incoming ties; repeaters, with both incoming and outgoing ties and sources, with only outgoing ties.

In our analysis, children are classified according to four profiles:

1. isolated and invisible, grouped together in December (similar to rejected and neglected)

2. sinks, more sociable (similar to popular)

3. repeaters, with an almost equal number of ties to and from the nodes (similar to average)

4. sources, the 'reference points', more often contacted by others than contacting others (similar to popular).

In comparison with the cognitive approach based on interview, our method allows to better specify popular sociometric status, splitted into sinks and sources.

The results of October and December show:

1. widespread aggressiveness of average children in October becomes sociability in December

2. $93 \%$ of the average of October becomes repeaters in December

3. popular pupils in October specialize their role, becoming sources or sinks in December.

Overall, the new definitions of role are clearly oriented towards an increase of sociability and specialization.

NA methods permit to combine these results, related to individual capabilities, with findings linked to relational dynamics arising from the network structure. As a consequence, the second criterion to analyse the relational competence is the lambda set. This procedure employs a partition criterion based on the greater or lesser capability of the subjects to be 'intermediaries'. We prefer this tool to the betweenness centrality measure because lambda set analysis is suitable for the immediate identification of three groups of pupils for each month, with the following relational traits:

1. connectors, supporting the whole net structure

2. bridges, intermediaries between groups or individuals

3. marginal, playing no strategic role as intermediaries.

Many nodes move from a group to another, causing an increase in the number of bridges in December, a weak decrease of connectors and a substantial decrease of marginal nodes. Only marginal nodes tend to remain in the same group: children, unable to be intermediaries in October, will rarely become in December.

As a third criterion, in order to select groups of children we employ cliques. This procedure identifies subjects with a good ability to build cohesive subgroups. In October the number of cliques is negligible: only 17 nodes show relational competence. It is, therefore, impossible to identify subgroups in the first month. In December, by contrast, the structure becomes more complex (Figure 3).

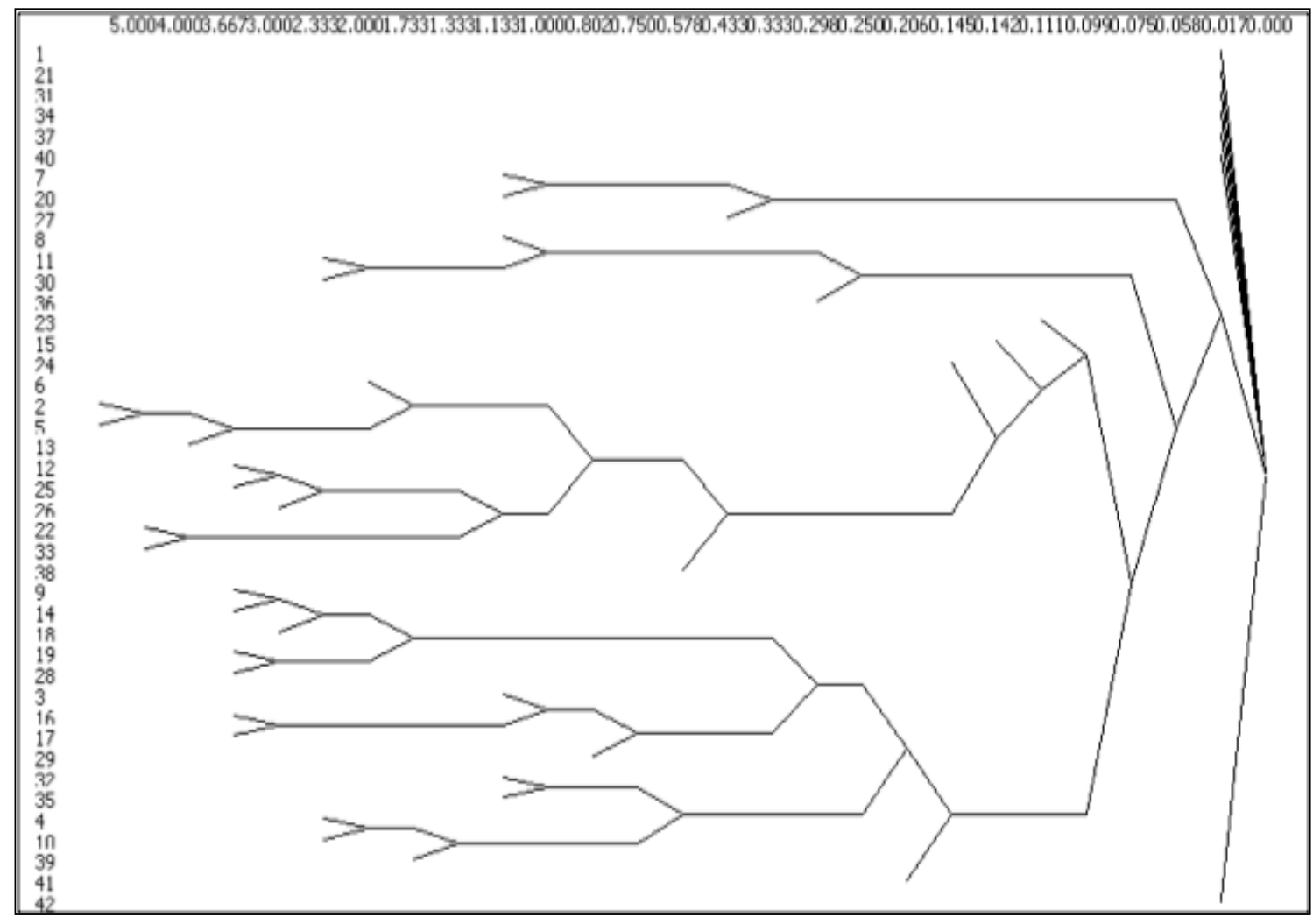

Figure 3. The dendrogram of the cliques of December. 
Finally, we obtain three groups:

1. embedded, children located in the majority of overlapping cliques, thanks to their ability to develop closed subgroups within the net

2. integrated, children with the ability to be part of simple, small groups, consisting of at least a triad

3. marginals, with weak capacity to belong to a group.

Once again, observing data from October to December, the number and profile of the groups in the network change while the relational competence increases.

\subsection{Relational Dynamics and the Criterion of Role}

As further step in our analysis, we study the association between individual characteristics and social roles. For example, what does to be popular in October mean? Can we detect a relationship between the underlying dynamics and those affecting sources or sinks in December? To answer this question, we employ the Kruskal-Wallis test combined with the Median test, introducing three blocks of variables (Table 1): structural attributes, sociability indicators and ego-network measures, the latter referred to links to and from each node but not to the whole network.

Table 1. Variables employed for Kruskal-Wallis test and Median test.

\begin{tabular}{|c|c|}
\hline Label & Description \\
\hline \multicolumn{2}{|r|}{ Variables } \\
\hline Age3, Age4, Age5 & Dichotomous variables: age \\
\hline GenderM, GenderF & Dichotomous variables: gender \\
\hline Brother1, brother2 & Dichotomous variables: number of siblings \\
\hline Cry & Dichotomous variable: propensity to cry \\
\hline Family Status: low, medium, high & Dichotomous variables: social class based to the parents' job \\
\hline \multicolumn{2}{|r|}{ Indicators of sociability } \\
\hline Pos_rel & Number of positive relationships \\
\hline Neg_rel & Number of negative relationships \\
\hline Index_Social impact & Index of social impact: total number of contacts per node \\
\hline Index Social preference & Index of social preference: difference between positive and negative relationships \\
\hline Index_Social aggressiveness & Index of social aggressiveness: difference between negative and positive relationships \\
\hline First_impact & Dichotomous variable: value 0 if the child has already been entered previously in school \\
\hline$\alpha$-parameter & Parameter detected by the $p^{l}$ model estimating the sociability as out-degree of each node \\
\hline$\beta$-parameter & Parameter detected by the $p^{l}$ model, estimating the attractiveness as in-degree of each node \\
\hline \multicolumn{2}{|r|}{ Measures of ego-network } \\
\hline EffSize & $\begin{array}{l}\text { Difference between the number of nodes with whom ego has ties and average number of links that } \\
\text { each of nodes has with other nodes: overall impact of the ego in the network }\end{array}$ \\
\hline Efficiency & $\begin{array}{l}\text { Relationship between EffSize and the size of the ego-network: impact of the ego on the network for } \\
\text { each new tie }\end{array}$ \\
\hline Size & Size of the network of ego: number of nodes (subjects) related to ego \\
\hline Ties & Number of ties of the ego \\
\hline Ordered pairs & Number of possible ties of the ego \\
\hline Density & Percentage of ties observed over the total number of possible ties \\
\hline nBroker & $\begin{array}{l}\text { Normalised brokerage capacity, i.e. ability of mediation of the ego (measured by the number of pairs } \\
\text { of neighbours not directly connected to each other divided by the total number of possible pairs, to } \\
\text { remove the effect of the size of the network) }\end{array}$ \\
\hline$n E g o B e$ & $\begin{array}{l}\text { Normalised Betweenness: capacity of each ego to connect subjects/groups each other compared with } \\
\text { the total capacity to connect subjects/groups in the network (Betweenness of the whole network) }\end{array}$ \\
\hline
\end{tabular}


The non-parametric tests are based on the same assumptions and often produce very similar results. The Median test is employed to assess the impact of each relational attribute. With this aim, we check the significance of each attribute referred to every cluster, identified by means of regular equivalence, lambda sets and cliques.

Further information concerning each child is obtained by means of a $p^{l}$ model [29], estimating the presence of a tie on the basis of two global parameters ( $\theta$-parameter as density and $\rho$-parameter as reciprocity level of network, respectively) and two individual ones ( $\alpha$-parameter as sociability and $\beta$-parameter as attractiveness of each node, respectively).

In our analysis, we use only the individual parameters, since they define each node by means of his position within the whole network. The two individual $\alpha$ - and $\beta$-parameters, however, are estimated to explain the presence of links, calculating the expected values of all the links between the various pairs of nodes.

In line with our research hypothesis, we assume that some relational dynamics observed in October contribute to define December's social roles.
Identifying the roles of isolated, invisible, average and popular by regular equivalence, the attributes significantly associated with each role are: positive or negative relationships and indices of social impact and social preference.

Since in October the network is building, the ego-measures related to the roles are fewer than in December (Table 2). In October EffSize, Size, Pairs, $n E g o B e$ measures and the $\alpha$-parameter are statistically significant as estimations of sociability for each node. In addition, in December even Ties, Density and nBroke are statistically significant, while only $n E g o B e$ is not. This means that in the last month the capability to be intermediary in the whole net (nBroke) is more important than to be bridge within the own ego-network ( $\mathrm{Eg} g \mathrm{Be}$ ). As a consequence, the unique feature of October significantly associated with the social roles emerging in December is the propensity to be connectors, defined by lambda sets. This affects, then, the arising of the different roles of isolated and invisible, sinks, repeaters and sources in the last month.

Table 2. Kruskal-Wallis test: measures by regular equivalence.

\begin{tabular}{|c|c|c|}
\hline Roles by structural equivalence & October $\chi^{2}$ & December $\chi^{2}$ \\
\hline Age3 & 3,65 & 1,01 \\
\hline Age4 & 0,62 & 4,12 \\
\hline Age5 & 2,71 & 2,28 \\
\hline GenderM & 4,81 & 1,4 \\
\hline Brother0 & 0,42 & 1,82 \\
\hline Brotherl & 2,98 & 4,21 \\
\hline Brother2 & 2,34 & 3,41 \\
\hline Cry & 1,04 & 1,62 \\
\hline StatusLow & 1,88 & 5,86 \\
\hline StatusMedium & 1,39 & $8,18^{*}$ \\
\hline StatusHigh & 0,84 & 4,07 \\
\hline Pos_rel & $24,17 * * *$ & $18,71 * * *$ \\
\hline Neg_rel & $13,8^{* *}$ & $23,46^{* * *}$ \\
\hline Index_Social impact & $31,9 * * *$ & $29,47 * * *$ \\
\hline Index_Social preference & $10,62 *$ & 3,17 \\
\hline Index_Social aggressiveness & $10,62 *$ & 3,17 \\
\hline First_impact & 1,67 & 3,83 \\
\hline EffSize & $18,55 * * *$ & $11,1^{*}$ \\
\hline Efficiency & 4,68 & 0 \\
\hline Size & $17,6^{* *}$ & $13,29 * *$ \\
\hline Ties & 6,897 & $17,86^{* * *}$ \\
\hline Ordered pairs & $17,6^{* *}$ & $13,29 * *$ \\
\hline Density & 2,72 & $13,38^{* *}$ \\
\hline nBroker & 2,3 & $13,72 * *$ \\
\hline$n E g o B e$ & $13,87 * *$ & 3,24 \\
\hline Alpha & $30,35 * * *$ & $17,47^{* *}$ \\
\hline Beta & 3,01 & 2,36 \\
\hline Isolated Oct & & 6,02 \\
\hline Invisible Oct & & 1,48 \\
\hline Average Oct & & 1,21 \\
\hline Popular Oct & & 6,27 \\
\hline Lambda: Connectors Oct & & $8,82 *$ \\
\hline Lambda: Bridges Oct & & 3,17 \\
\hline Cliques Oct & & 2,94 \\
\hline
\end{tabular}

\footnotetext{
* significant for $\alpha=0.05$; ** significant for $\alpha=0.01$; *** significant for $\alpha=0.001$.
} 

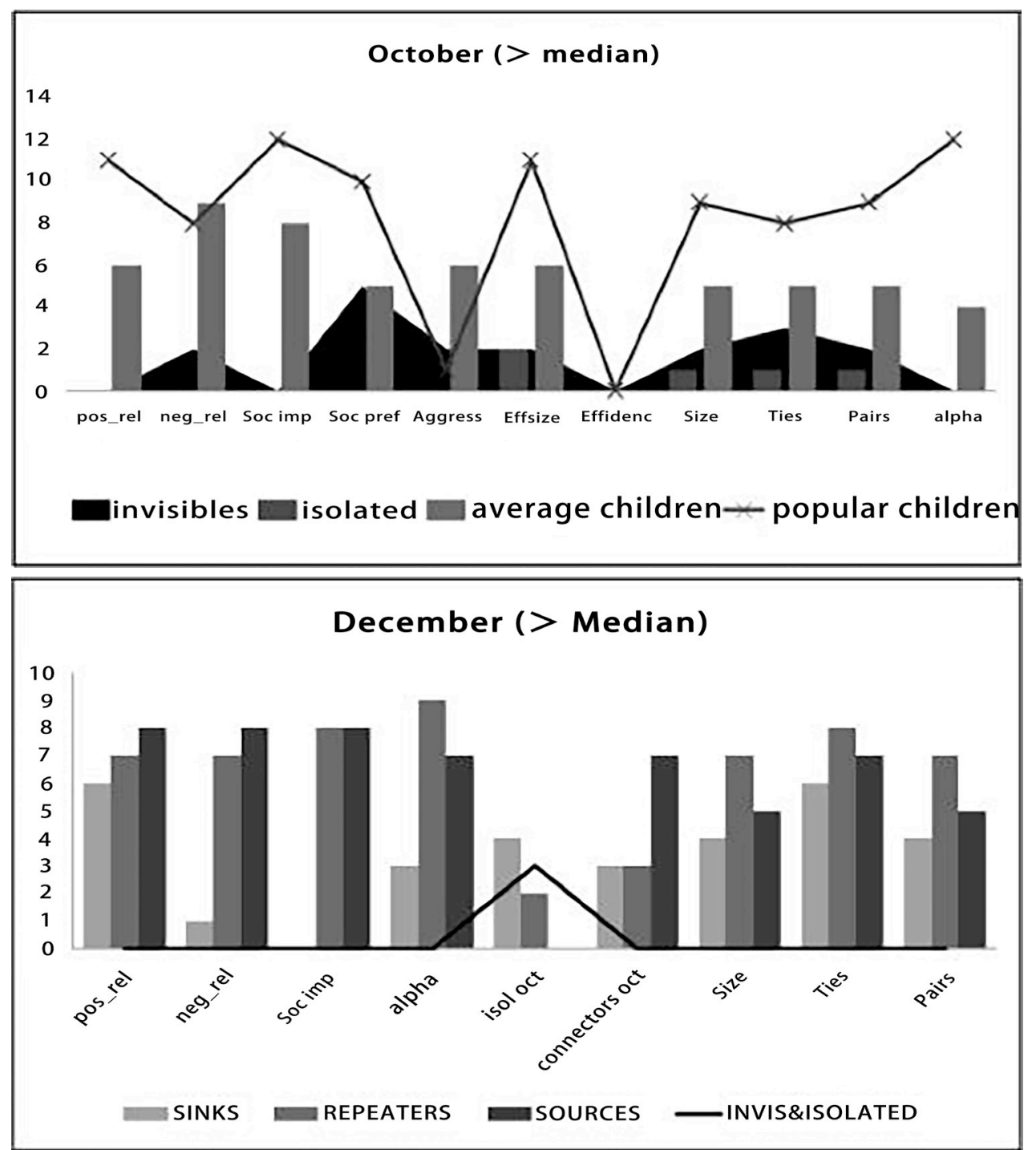

* The figure shows only the significant values according to the Median test.

Figure 4. Median test: weights of significant measures related to the roles (regular equivalence)*.

Overall, from October to December, the number of significant values decreases referred to the indicators of sociability (social impact, preferences and aggressiveness) and increases for individual attributes and ego-network measures. These findings are more affected by structural and relational dynamics than by individual features, as only NA methods allow to highlight.

Since, looking at the Kruskal-Wallis test of December, the only significant cluster defined in October is connectors (lambda_connectorsOct), the role configuration entirely changes. Over time we observe changes in the roles, such as from average to repeater and a specialization from popular to source or sink, both increasing in sociability. Isolated and invisible, indeed, almost completely disappear.

Employing the Median test, we obtain the weights of each significant indices related to the roles (Figure 4).

In October, in terms of sociability, popular children have the highest propensity to form positive relationships. Both popular and average pupils have a high social impact while social preference is limited among average, similar to invisible. Initially, the aggressiveness is widespread in the modal category of average, while the lowest level is recorded for popular. In October, the aggressiveness of average is likely due to the first impact with the school setting. Invisible and isolated children are the least sociable.

In December, by means of REGE algorithm, we select two new groups, namely sinks and sources. The sinks have very few negative contacts while the sources have the highest propensity to contact by both positive and negative relationships. The popular children of October become sinks and sources in December. Sources and repeaters have the greatest social impact on the network. In terms of the $\alpha$-parameter, repeaters are the most sociable children.

Through the ego-network measures (EffSize, Efficiency, Size, Ties, Pairs, $\alpha$-parameter), the results of October are predictable: popular children show higher values than other 
roles for all significant indices, followed by average, invisible and isolated pupils. In December, repeaters have the highest values, followed by sources and sinks.

The underlying dynamics involved represent an especially significant aspect of our study. The repeaters of December, in fact, are mostly average children in October. Besides, from October to December, pupils improve their ability as intermediaries at least within their own ego-networks, whereas it is more difficult to play this role if the network is considered as a whole.

\subsection{Relational Dynamics and Brokerage}

The lambda set procedure draws clusters of pupils focusing on the ability to act as brokers. Three groups of children are observed, namely:

1. connectors, supporters of the structure of the network

2. bridges, intermediaries between groups or individuals

3. marginal nodes.

In December, the number of bridges increases while marginal pupils decrease much more than connectors do. By means of the Kruskal-Wallis test (Table 3), the main differences are in the sociability indicators. Compared with October, in December, aggressiveness and a larger number of positive contacts rather than negative are not associated with the ability to act as intermediary. However, both positive and negative relationships are equally associated with the broker ability. Besides, the most of ego-measures are significant in October but only few are still significant in December: only ego-measures showing the subjective ability to relate to others (Size, Ties, Pairs, Ego-Betweeness) retain their significance.

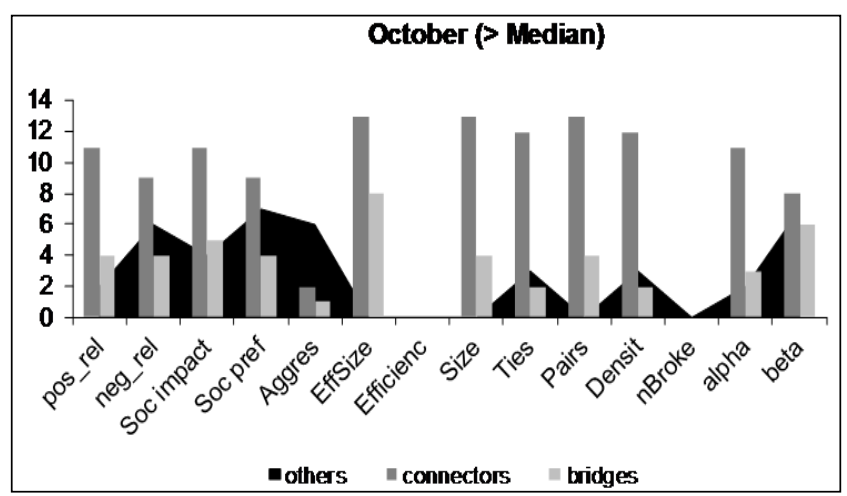

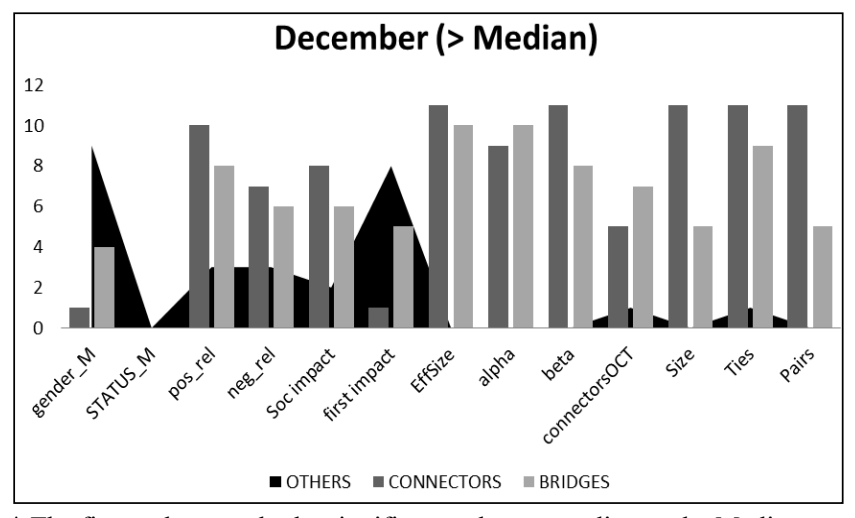

* The figure shows only the significant values according to the Median test Figure 5. Median test: weights of significant measures related to the roles (lambda sets)*.

Finally, most of October's connectors become bridges or keep their role in December. Individual sociability, measured by $\alpha$-parameter, and attractiveness, by $\beta$-parameter, show really impressive trends from the first to the other month (Figure 5).

Summing-up, in October bridges and others have quite similar ego-network values. Connectors, instead, show the highest values for most of indices and, in particular, they are both the most sociable and attractive pupils. The dynamics of December are different. Namely, connectors are the most attractive ( $\beta$-parameter) and bridges are the most sociable $(\alpha$-parameter).

\subsection{Relational Dynamics and the Ability to belong to Subgroups}

The ability to build group relationships is analysed by means of cliques, which focuses on the presence of groups among children. This dynamic is certainly the most influenced by time. The difference is so great that, while in October it is possible to find only 17 children belonging to a group, in December we distinguish two clusters: embedded and integrated.

Analysing the values of the measures (Table 4), the presence of positive relationships and indices of preference and aggressiveness are relevant in October, while in December there is a positive association with social impact and first school entrance and also with the values of $\alpha$ - and $\beta$-parameters. Focusing on the ego-networks measures Size, Ties, Pairs, Density, Efficiency and Nbroker - are significantly associated with the unique group drawn in October, while in December the significance holds only for the Efficiency. 
Table 3. Kruskal-Wallis test: role of 'broker' by (lambda sets).

\begin{tabular}{|c|c|c|}
\hline Roles by lambda sets & October $\chi^{2}$ & December $\chi^{2}$ \\
\hline Age3 & 0,55 & 2,28 \\
\hline Age4 & 1,23 & 2,11 \\
\hline Age5 & 1,54 & 0,2 \\
\hline GenderM & 0,72 & $8,91 * *$ \\
\hline Brother0 & 0,4 & 0,58 \\
\hline Brother1 & 1,22 & 1,23 \\
\hline Brother2 & 2,1 & 2,31 \\
\hline Cry & 1,49 & 2,11 \\
\hline StatusLow & 4,39 & 3,44 \\
\hline StatusMedium & 3,14 & 5,55 \\
\hline StatusHigh & 0,52 & 1,86 \\
\hline Pos_rel & $18,45 * * *$ & $12,35^{* *}$ \\
\hline Neg_rel & 2,82 & $9,01^{*}$ \\
\hline Index_Social impact & $11,17^{* *}$ & $15,32 * * *$ \\
\hline Index_Social preference & $6,27^{*}$ & 0,771 \\
\hline Index_Social aggressiveness & $6,27^{*}$ & 0,771 \\
\hline First_impact & 2,46 & 5,51 \\
\hline EffSize & $33,57 * * *$ & $34,96 * * *$ \\
\hline Efficiency & $15,02 * *$ & 0 \\
\hline Size & $34,15 * * *$ & $36,68 * * *$ \\
\hline Ties & $24,04 * * *$ & $26,09 * * *$ \\
\hline Ordered pairs & $34,15^{* * *}$ & $36,68^{* * *}$ \\
\hline Density & $10,02 * *$ & 1,18 \\
\hline nBroker & $9,45 * *$ & 1,47 \\
\hline nEgoBe & 0,264 & $6,43^{*}$ \\
\hline Alpha & $16,346^{* * *}$ & $21,39 * * *$ \\
\hline Beta & $10,214 * *$ & $23,53 * * *$ \\
\hline Isolated Oct & & 1,88 \\
\hline Invisible Oct & & 0,991 \\
\hline Average Oct & & 0,888 \\
\hline Popular Oct & & 3,221 \\
\hline Lambda: Connectors Oct & & $6,29 *$ \\
\hline Lambda: Bridges Oct & & 2,68 \\
\hline Cliques Oct & & 0,49 \\
\hline
\end{tabular}

* significant for $\alpha=0.05$; ** significant for $\alpha=0.01$; *** significant for $\alpha=0.001$. 
Table 4. Kruskal-Wallis test: measures by cliques.

\begin{tabular}{|c|c|c|}
\hline Roles by cliques & October $\chi^{2}$ & December $\chi^{2}$ \\
\hline Age3 & 0,112 & 4,01 \\
\hline Age4 & 0,006 & 4,84 \\
\hline Age5 & 0,24 & 0,3 \\
\hline GenderM & 0,003 & $6,81^{*}$ \\
\hline Brother0 & 0,004 & 0,04 \\
\hline Brother1 & 0,19 & 1,61 \\
\hline Brother2 & 0,26 & 2,14 \\
\hline Cry & 0,067 & 0,36 \\
\hline StatusLow & 0,073 & 3,45 \\
\hline StatusMedium & 0,617 & 2,57 \\
\hline StatusHigh & 0,898 & 2,899 \\
\hline Pos_rel & $6,689^{*}$ & $20,78 * * *$ \\
\hline Neg_rel & 0,228 & 7,31 \\
\hline Index_Social impact & 3,16 & $16,74 * * *$ \\
\hline Index_Social preference & $4,96^{*}$ & 4,17 \\
\hline Index_Social aggressiveness & $4,96^{*}$ & 4,17 \\
\hline First_impact & 0,05 & $6,74 *$ \\
\hline EffSize & $5,27 *$ & $17,87 * * *$ \\
\hline Efficiency & $25,04 * * *$ & 0 \\
\hline Size & $7,18^{*}$ & 0 \\
\hline Ties & $22,75^{* * *}$ & 0 \\
\hline Ordered pairs & $7,18^{*}$ & 0 \\
\hline Density & $25,71 * * *$ & 0 \\
\hline nBroker & $26,5^{* * *}$ & 0 \\
\hline nEgoBe & 0,018 & 0 \\
\hline Alpha & 3,03 & $18,096^{* * *}$ \\
\hline Beta & 0,914 & $18,209 * * *$ \\
\hline Isolated Oct & & 0,562 \\
\hline Invisible Oct & & 1,45 \\
\hline Average Oct & & 3,83 \\
\hline Popular Oct & & 1,831 \\
\hline Lambda: Connectors Oct & & 2,642 \\
\hline Lambda: Bridges Oct & & 1,066 \\
\hline Cliques Oct & & 2,63 \\
\hline
\end{tabular}

* significant for $\alpha=0.05 ; * *$ significant for $\alpha=0.01 ; * * *$ significant for $\alpha=0.001$. 
In October the members of cliques show a particularly high propensity to build positive ties, while the relevance of Size, Ties, Pairs, Density, EffSize, as well as the low aggressiveness, highlights the relevant social competence of this group (Figure 6).
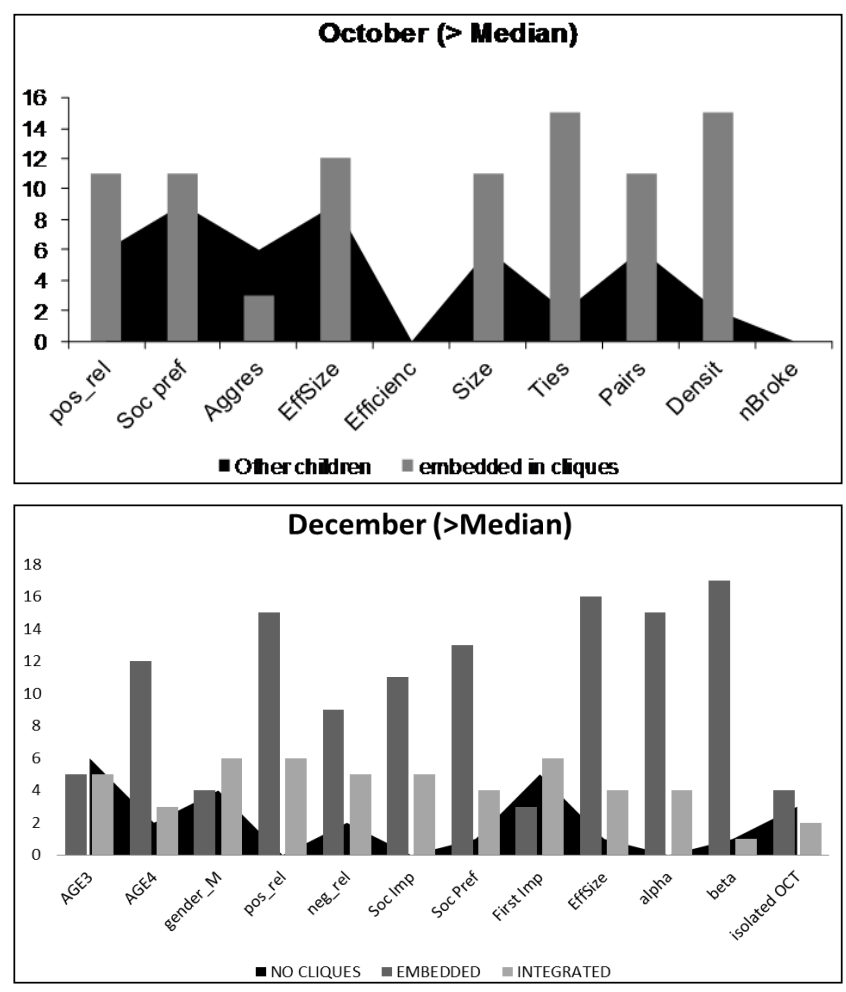

* The figure shows only the significant values according to the Median test.

Figure 6. Median test: weights of significant measures related to the roles $(\text { cliques })^{*}$

In December, moreover, a difference between embedded and integrated children arises. The embedded are those who have a strong propensity to belong to groups. These pupils have the highest values for almost all computed measures. They build many positive relationships rather than negative ones. They are reference points ( $\beta$-parameter) for others and are also sociable ( $\alpha$-parameter). They, indeed, contact other pupils but are also often contacted by others. The integrated pupils have quite equal positive and negative ties. Furthermore, they have a higher propensity to sociability $\alpha$-parameter rather than to be reference points $\beta$-parameter.

\section{Conclusions}

In this paper the development of social roles among children, focusing on peer-to-peer relationships, is analysed by means of NA methodology. The aim is both to detect the presence of social roles and explore the structure of the relationships among children. We carried out our research employing the method of direct observation since at this age there is a considerable gap between verbal and relational competence.

In our findings, even if the networks of October and
December are really different, we detect a coherent evolution in the relational structure and role building. The network of December shows an increasing relevance of brokers' and reference points or popular children. We notice only few isolated children and high levels of interaction among pupils. This implies an increasing specialization in the individual roles even among children aged 3-5.

NA methods, notably lambda sets and cliques, allows detecting suggestive relational dynamics, not necessarily similar to the traditional sociometric status. Only the regular equivalence procedure, instead, provides similar results to those already known in literature.

\section{REFERENCES}

[1] J. Perner, T. Ruffman, S. R. Leekam. Theory of mind is contagious: you catch it from your sibs, Child Development, 65, 1228-1238, 1994.

[2] C. Hughes, J. Dunn. Pretend you didn't know»: Preschoolers' talk about mental states in pretended play, Cognitive Development, No. 12, 381-403, 1997.

[3] A. J., Santos, J.R. Daniel, C. Fernandes, B. E. Vaughn. Affiliative Subgroups in Preschool Classrooms: Integrating Constructs and Methods from Social Ethology and Sociometric Traditions. PLoS ONE 10(7): e0130932. doi:10.1371/journal.pone.0130932, 2015.

[4] S. F. Nadel. The Theory of Social Structure, London: Cohen \&West, 1957.

[5] R. K. Merton. Social Theory and Social Structure, 2nd edition, Glencoe: Free Press, 1959.

[6] J. D. Coie, K. A. Dodge, H. Coppotelli. Dimensions and types of social status in the school: A cross-age comparison, Developmental Psychology, No. 18, 557-570, 1982.

[7] K. A. Dodge, D. C. Schlundt, I. Schocken, J. D. Delugach. Social competence and children's sociometric status: the role of peer group entry strategies, Merrill-Palmer Quarterly, 29, 309-336, 1983.

[8] J. D. Coie, K. A. Dodge, J. B. Kupersmidt. Peer group behaviours and social status, in S. R. Asher, J. C. Coie Eds., Peer rejection in childhood, New York, NY: Cambridge University Press, 17-59, 1990.

[9] A. F. Newcomb, W. M. Bukowski, L. Pattee. Children's peer relations: A meta-analytic review of popular, rejected, neglected, controversial, and average sociometric status, Psychological Bulletin, No. 113, 99-128, 1993.

[10] J. W. Maag, S. F. Vasa, R. Reid, G. K. Torrey. Social and behavioural predictors of popular, rejected and average children, Educational and Psychological Measurement, No. 552, 196-205, 1995.

[11] J. D. Coie, K. A. Dodge. Continuities and changes in children's social status: A five-year study, Merrill-Palmer Quarterly, No. 29, 261-282, 1983.

[12] J. D. Coie, K. A. Dodge. Multiple sources of data on social 
behaviour and social status in the school: A cross-age comparison, Child Development, No. 593, 815-829, 1988.

[13] A. F. Newcomb, W. M. Bukowski. Social impact and social preference as determinants of children's peer group status, Developmental Psychology, No. 19, 856-867, 1983.

[14] R. Veenstra, J. K. Djikstra, C. Steglich, M. H. W. Van Zaik (Eds.). Special issue: Network and behavior dynamics in adolescence. Journal of Research on Adolescence, 23, 399412. doi: 10.1111/ jora.12070, 2013.

[15] S. D. Gest, A. J. Davidson, K. L. Rulison, J. Moody, J. A. Welsh. Features of groups and status hierarchies in girls' and boys' early adolescent peer networks. New Directions for Child and Ado- lescent Development, 43-60. doi: 10.1002/cd.200, 2007.

[16] A. J. Santos, B. E. Vaughn, K. K. Bost. Specifying social structures in preschool classrooms: descriptive and functional distinctions between affiliative subgroups. Acta Ethologica, 11, 101-113. doi: 10.1007/s10211-008-0047-0, 2008.

[17] C. J. Patterson, J. B., Kupersmidt, P. C. Griesler. Children's perceptions of self and of relationships with others as a function of sociometric status, Child Development, No. 61, $1335-1349,1990$

[18] D. R. Schaefer, J. M. Light, R. A. Fabes, L. D. Hanish, C. L. Martin. Fundamental Principles of Network Formation among Preschool Children, Social Networks, No. 321, 61-71, 2010.

[19] R. Boudon. Effets pervers et ordre social, Paris: PUF, 1977.

[20] F. Lorrain, H. C. White. Structural equivalence of individuals in social networks, Journal of Mathematical Sociology, No. 1, 67-80, 1971 .

[21] M. G. Everett. Role similarity and complexity in social networks, Social Networks, No. 7, 353-359, 1985.

[22] S. P. Borgatti, M. G. Everett. Two algorithms for computing regular equivalence, Social Networks, No. 15, 361-376, 1993.

[23] K. Faust. Comparison of Methods for Positional Analysis: Structural and General Equivalences, Social Networks, No. 10, 313-341, 1988.

[24] D. R. White, K. P. Reitz. Graph and semigroup homomorphisms on networks of relations, Social Networks, No. 6, 193-234, 1983.

[25] S. P. Borgatti, M. G. Everett, P. R. Shirey. LS sets, lambda sets and other cohesive subsets, Social Networks, No. 12, 337-357, 1990.

[26] R. Luce, A. Perry. A method of matrix analysis of group structure, Psychometrika, No. 14, 95-116, 1949.

[27] C. Bron, J. Kerbosch. Finding all cliques of an undirected graph, Communications of the ACM, No. 169, 575-577, 1973.

[28] D. Hansen, B. Shneiderman, M. A. Smith. Analyzing Social Media Networks with NodeXL: Insights from a Connected World. Burlington, MA: Morgan Kaufmann-Elsevier, 2011.

[29] P. W. Holland, S. Leinhardt. An Exponential Family of Probability Distributions for Directed Graphs, Journal of the American Statistical Association, Vol. 76, No. 373, 33-50, 1981 